\title{
Study on the Method of Detecting Phycocyanin by Fluorescence Spectroscopy
}

\author{
Jinxia Wang, a , Le Luo ${ }^{1, b}$, Lingling Zhan ${ }^{1, c}$ \\ ${ }^{1}$ Chongqing Vocational Institute of Engineering, Chongqing, China

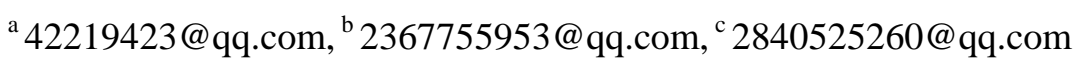

Keywords: fluorescence spectrum; phycocyanin; cyanobacteria; biomass

\begin{abstract}
The phycocyanin is a unique protein of cyanobacteria. In this study, fluorescence spectroscopy was used to study the algae of Anabaena sinensis. Firstly, the optimal detection conditions were determined by fluorescence scanning of living algae cells: $\lambda \mathrm{ex}=620 \mathrm{~nm}$, $\lambda \mathrm{em}=650 \mathrm{~nm}$. The relationship between the fluorescence intensity of phycocyanin and the biomass of algal cells was established $(\mathrm{R} 2=0.9972)$. The method was applied to the study of cyanobacterial growth. The results showed that the method has high precision (RSD=3.32\%) and repeats. Sex $(\mathrm{RSD}=3.87 \%)$ is good, and the sample does not need to be treated.
\end{abstract}

\section{Introduction}

With the increasing eutrophication of water bodies, pollution incidents caused by toxic cyanobacteria bloom frequently occur worldwide, and the toxins produced by them are potentially harmful to human health, and environmental and economic problems are causing people's attention $^{[1] \text {. }}$

At present, the detection of cyanobacterial biomass mostly uses the method of algae cell counting and chlorophyll a determination, but the algae microscopic counting method is inefficient, and the experience of the analyst is high but the precision is low. The method for detecting chlorophyll a is not specific and cannot be The distinction between phytoplankton and cyanobacteria requires a lot of manpower and material resources. Therefore, it is urgent to find a fast and accurate cyanobacteria biomass detection technology.

Fluorescence spectroscopy has the advantages of high sensitivity, no need for tedious pretreatment of the sample and no damage to the sample structure [2-4]. At present, various fluorescence spectroscopy techniques such as fluorescence excitation spectroscopy, fluorescence emission spectroscopy, simultaneous fluorescence spectroscopy, and three-dimensional fluorescence spectroscopy are widely used to qualitatively or quantitatively describe the physicochemical properties of organic matter.

The phycocyanin is a pigment unique to cyanobacteria, so the amount of phycocyanin can reflect the biomass of cyanobacteria to a certain extent. The phycocyanin is mainly a porphyrin pigment protein with strong fluorescent properties, so it can be detected by algae. The cytoplasmic fluorescence intensity responds rapidly and efficiently to the biomass of cyanobacteria. In this study, the fluorescence excitation wavelength and emission wavelength of phycocyanin were determined by scanning the fluorescence spectrum of Anabaena sinensis. The fluorescence intensity of phycocyanin was detected by fluorescence spectroscopy, and the cyanobacterial biomass and phycocyanin characteristic fluorescence were established. Detection relationship. A simple and quick method for determining the biomass of cyanobacteria in water.

\section{Materials and Methods}

The selected algae species is Anabaena flos-aquae1092 in cyanobacteria, which is derived from the Algae Collection Center of the Institute of Hydrobiology, Chinese Academy of Sciences. After activation, the cells were cultured in a constant temperature light incubator at a culture temperature of $25^{\circ} \mathrm{C}$, a light intensity of 2000 to 2500 lux, and a light-dark cycle ratio of $14 \mathrm{~h}$ : $10 \mathrm{~h}$, and cultured using a CT culture solution. 
The main application was measured by F-7000 fluorescence spectrophotometer (Japan Hitachi, Ltd.), and a few algae liquid was added to a $50 \mathrm{~mL}$ standard test tube, shaken, and transferred to a 1 $\mathrm{cm}$ quartz cuvette for measurement by fluorescence spectroscopy. Measurement conditions: slit 5 $\mathrm{nm}$, scanning speed $2400 \mathrm{~nm} / \mathrm{min}$, excitation wavelength $(\lambda$ ex) $500-700 \mathrm{~nm}$, emission wavelength $(\lambda \mathrm{em}) 500-700 \mathrm{~nm}$, step size $5 \mathrm{~nm}$, voltage $700 \mathrm{~V}$, scanning phycocyanin three-dimensional fluorescence spectrum; Good $\lambda \mathrm{ex} / \lambda \mathrm{em}$. The quantitative detection of the sample was carried out in the photometric mode with the best $\lambda$ ex/ $/ \lambda$ em obtained.

\section{Results and Analysis}

Fig. 1 is a two-dimensional fluorescence spectrum of the phycocyanin of the genus Anabaena, the abscissa is the emission wavelength $\lambda \mathrm{em}$, the ordinate is the excitation wavelength $\lambda$ ex, and the contour line indicates the fluorescence intensity. Fig. 2 is a three-dimensional fluorescence spectrum of phycocyanin. As can be seen from Fig. 1 and Fig. 2, there is a linear fluorescent band on the upper left side of the fluorescence image, which is a Raman scattering region of water ${ }^{[5]}$. The position of this type of scattering effect in the three-dimensional fluorescence spectrum is relatively fixed and does not affect the experimental analysis. The results showed that the peak of phycocyanin appeared at 620/650 $\mathrm{nm}$. Some scholars have found that phycocyanin has the strongest fluorescence effect in the range of fluorescence excitation wavelength of $620 \mathrm{~nm}{ }^{[6]}$ and emission wavelength of $650-660 \mathrm{~nm}^{[7]}$. Therefore, the fluorescence excitation wavelength $\lambda \mathrm{ex}=620 \mathrm{~nm}$ and the emission wavelength $\lambda \mathrm{em}=650 \mathrm{~nm}$ were selected as the characteristic wavelengths for detecting the relative content of phycocyanin by fluorescence spectroscopy. This characteristic uses this characteristic wavelength to detect the characteristic fluorescence of cyanophycocyanin.

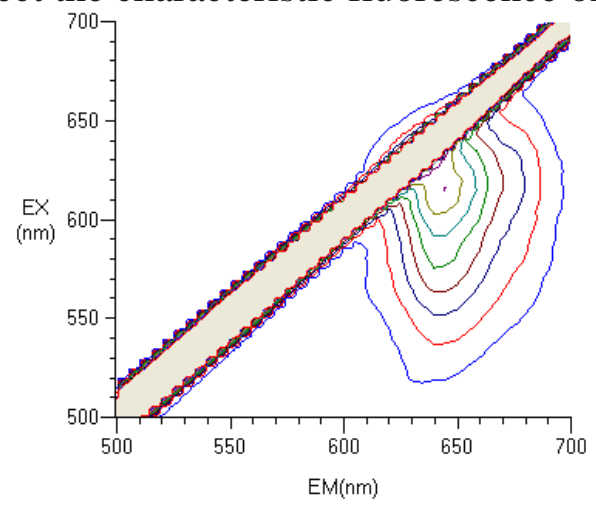

Fig.1 Two-Dimensional Fluorescence Spectrum of Phycocyanin

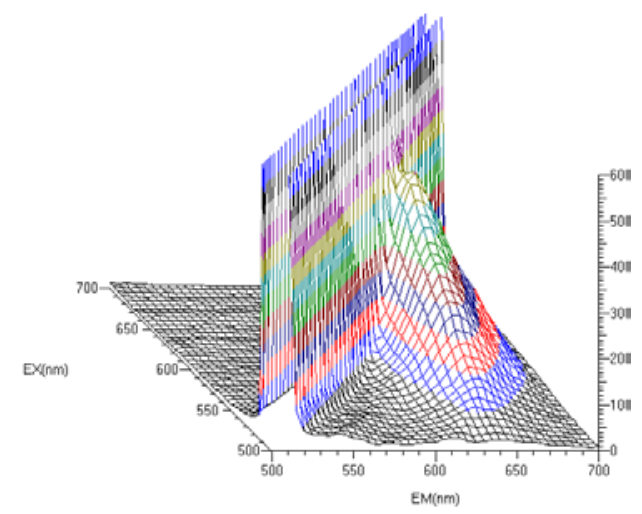

Fig.2 Three-Dimensional Fluorescence Spectrum of Phycocyanin

The fluorescence intensity of algal biomass and phycocyanin was measured at each concentration, and the relationship between algal biomass and characteristic fluorescence intensity was established. Figure 3 is the relationship between algal biomass and phycocyanin fluorescence intensity. It can be seen from Fig. 3 that the linear relationship between the concentration of algae cells and the characteristic fluorescence intensity of phycocyanin is good in the research range (the 
cell concentration range of $105-107$ cells $/ \mathrm{mL}$ ), $\mathrm{R} 2=0.9972$. It is further explained that its optimum $\lambda$ ex/ $\lambda$ em has reliability. It is proved that the selective detection of cyanobacterial biomass by fluorescence spectrometry is feasible.

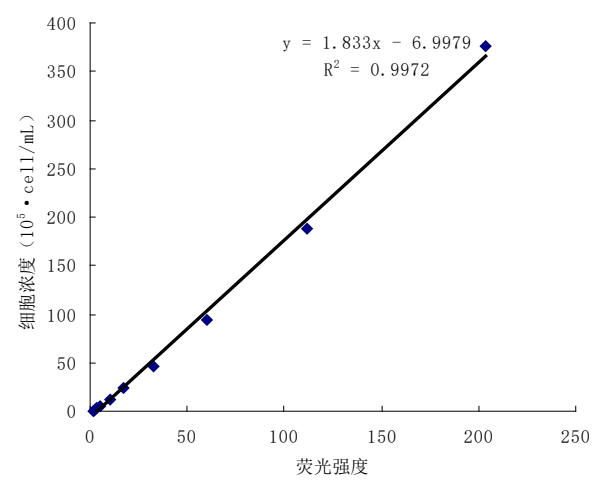

Fig.3 Relationship between Biomass and Fluorescence Intensity of Phycocyanin Characteristic

Take a certain amount of algae solution, shake it, and measure it continuously 7 times by fluorescence spectroscopy. Figure 4 is the fluorescence spectrum of the precision experiment. The fluorescence intensity is $106,109.9,111.5,116,116.3,113.7$ and 114.9 , respectively. 112.61 , the RSD is $3.32 \%$, and the experiment proves that the method has good precision.

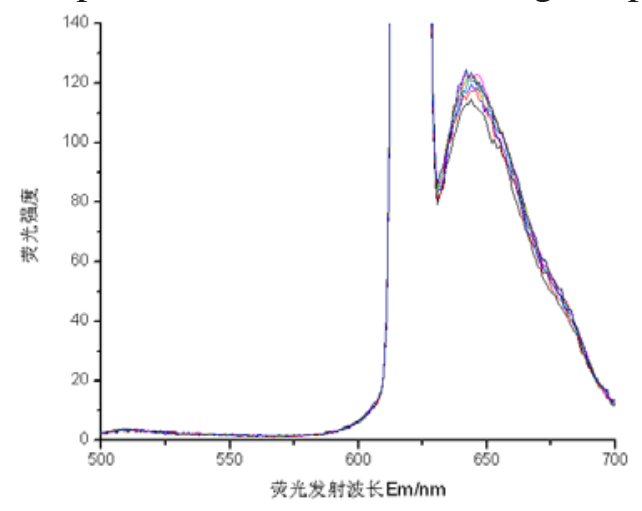

Fig.4 Fluorescence Spectrum of theprecsion test

Take 5 parts of the same algae solution and measure according to the above method. The fluorescence spectrum of the repeatability experiment is shown in Fig. 5. The fluorescence intensities are: 106, 111.8, 110.4, 115.6 and 116.9, the average value is 112.14, and the RSD is $3.87 \%$. The experiment proves that the method is heavy.

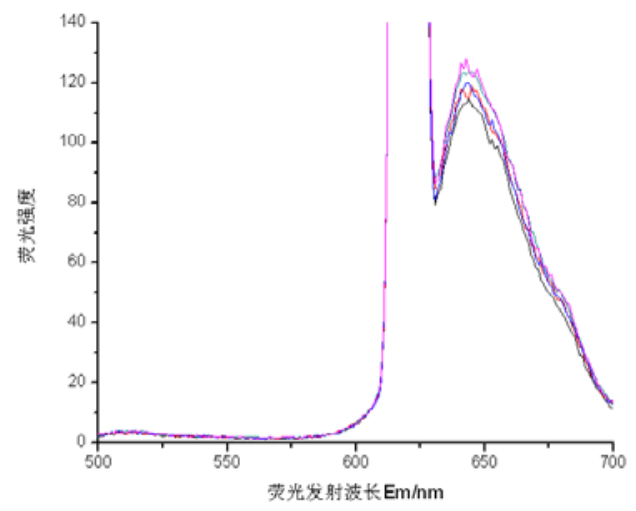

Fig.5 Fluorescence Spectrum of repetitive experiment

Under sterile conditions, $150 \mathrm{~mL}$ of algae solution was added to a $250 \mathrm{~mL}$ sterile flask to be cultured in a constant temperature light incubator at a temperature of $25^{\circ} \mathrm{C}$, a light intensity of 2000 to 2500 lux, and a light-dark cycle ratio of 14 h: 10 h. , to detect its characteristic fluorescence 
intensity. Set up 3 parallel samples and take the average. The test results are shown in Figure 6. Throughout the experiment, the results showed that the fluorescence intensity increased with the increase of algae cell number, from 60.3 on the first day to 171.7 on the seventh day. The fluorescence intensity of algae increased gradually, indicating that the number of algae cells and fluorescence intensity had A good linear growth relationship better reflects the growth of cyanobacteria. It is indicated that the biomass of cyanobacteria can be quickly and accurately detected by measuring the characteristic fluorescence intensity of cyanobacterial phycocyanin in actual water samples.

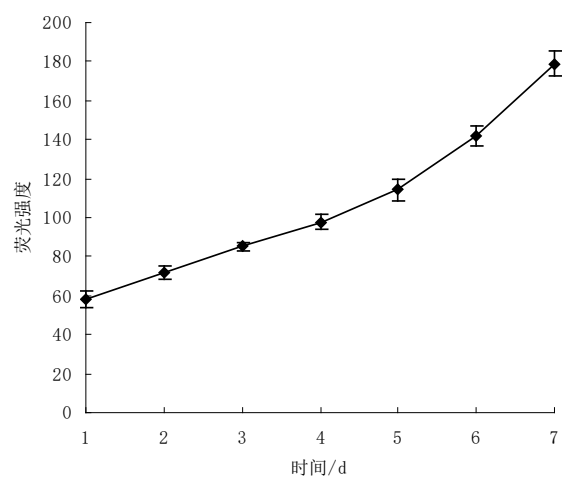

Fig.6 the growth condition of Anabaena flos-aquae

\section{Conclusion}

The optimal fluorescence excitation and emission wavelengths of phycocyanin in cyanobacteria were determined to be $620 \mathrm{~nm}$ and $650 \mathrm{~nm}$, respectively. The linear relationship between the biomass of Anabaena sinensis and the fluorescence intensity of phycocyanin was established. In actual detection, The biomass of cyanobacteria can be expressed by measuring the fluorescence intensity of phycocyanin, which has good precision and reproducibility. The method has the specificity and can distinguish the biomass of cyanobacteria from phytoplankton. Moreover, the sample does not need to be pretreated, and the algae liquid is directly tested, which simplifies the steps and provides a simple and quick new method for determining the cyanobacterial biomass measurement in the actual water sample.

\section{Acknowledgement}

Project Supported by Scientific and Technological Research Program of Chongqing Municipal Education Commission(Grant No. KJ1503302)

\section{References}

[1] Yao Yuling, Jiang Wenjing, Qu Qiu et al. Research progress on detection methods of microcystins in surface water and drinking water [J]. Modern Scientific Instruments, 2008, (5): 86-90.

[2] Liu Zhouyi, Zhu Tuo, Gu Endong et al.Study on Acidic Orange II by Fluorescence Spectroscopy[J].Acta Optica Sinica,2008,28(6):1106-1110.

[3] Liu Hailong, Wu Xijun, Tian Guangjun. Application of three-dimensional fluorescence spectroscopy and parallel factor analysis in green tea analysis and species identification [J]. China Laser, 2008, 35 (5): 685-689.

[4] Zhang Fang, Su Rongguo, Wang Xiulin, et al. Fluorescence characteristics extraction and identification measurement technology of phytoplankton [J]. China Laser, 2008, 35(12): 2052-2059.

[5] Jin Dan, Zhang Yuzhen, Li Guogang et al. Study on Three-Dimensional Fluorescence Spectra of 
Phenanthrene[J].Spectroscopy and Spectral Analysis, 2009,29(5): 1319-1322.

[6] Lee T-y, Tsuzuki M, Takeuchi T, et al. Quantitative determination of cyanobacteria in mixed phytoplankton assemblages by an in vivo fluorimetric method [J]. Analytica Chimica Acta, 1995, 302(1): 81-87.

[7] Beutler M, Wiltshire $\mathrm{KH}$, Arp $\mathrm{M}$, et al. A reduced model of the fluorescence from the cyanobacterial photosynthetic apparatus designed for the in situ detection of cyanobacteria [J]. Biochimica et Biophysica Acta, 2003, 1604(1): 33-46. 